\title{
CircRNA cRAPGEF5 is Downregulated in Gastric Cancer and Promotes the Transition from Precursor to Mature miR-22
}

\section{Yan Feng}

People's Hospital of Xinjiang Uygur Autonomous Region

\section{Chun Wang}

People's Hospital of Xinjiang Uygur Autonomous Region

\section{Tian Shi}

People's Hospital of Xinjiang Uygur Autonomous Region

\section{Weidong Liu}

people's hospital of xinjiang uygur autonomous

\section{Huan Liu}

People's Hospital of Xinjiang Uygur Autonomous Region

\section{Bo Zhu}

people's hospital of xinjiang uygur autonomous

Feng Gao ( $\sim$ FengGaoUrumqi@163.com )

People's Hospital of Xinjiang Uygur Autonomous Region

\section{Research article}

Keywords: cRAPGEF5, miR-22, precursor, proliferation

Posted Date: November 5th, 2020

DOl: https://doi.org/10.21203/rs.3.rs-101294/v1

License: (c) (i) This work is licensed under a Creative Commons Attribution 4.0 International License. Read Full License 


\section{Abstract}

Background: CircRNA cRAPGEF5 has been characterized as an oncogene in renal cell carcinoma. We in this study explored the involvement of cRAPGEF5 in gastric cancer (GC).

Methods: Expression of cRAPGEF5, mature miR-22 and miR-22 precursor in paired GC and non-tumor tissues was analyzed by RT-qPCR. Pearson's correlation coefficient was performed to analyze the correlations between them. GC cells were transfected with CRAPGEF5 expression vector, followed by measuring the expression levels of mature miR-22 and miR-22 precursor using RT-qPCR. Cell proliferation was analyzed by CCK-8 assay.

Results: We found that CRAPGEF5 was under-expressed in GC and positively correlated with mature miR22 , but not miR-22 precursor. In GC cells, CRAPGEF5 overexpression resulted in the upregulation of mature miR-22, but failed to significantly alter the expression of miR-22. Cell proliferation analysis showed that CRAPGEF5 and miR-22 overexpression decreased cell proliferation, while miR-22 inhibitor played an opposite role. Moreover, miR-22 overexpression attenuated the effect of CRAPGEF5 overexpression.

Conclusions: Therefore, CRAPGEF5 may promote the maturation of miR-22 to suppress GC cell proliferation.

\section{Background}

Gastric cancer (GC) is one of the most common types of malignancies with more than $90 \%$ of cases are adenocarcinoma.[1] It is estimated that GC affects more than 1 million people each year.[2] With timely and appropriate treatment, more than $70 \%$ of GC patients diagnosed with local tumors can survive 5 years.[3] However, tumor metastasis to distant sites, such as liver, peritoneum, lung, and bone, is common.[4] Once distant tumor metastasis has occurred, only less than $30 \%$ of GC patients can survive 5 years, mainly owing to the lacking of effective treatments. [5, 6] Infections of Helicobacter pylori, smoking and poor diet are the major risk factors for GC, while the molecular mechanism of GC remains poorly elucidated, which limits the development of novel anti-GC therapies.[7]

Previous studies of the molecular pathogenesis of GC have revealed a considerable number of molecular pathways involved in the development and progression of GC, such as STAT3 Pathway and Hedgehog signaling pathway.[8, 9] Some of the pathways have been proven to be promising targets for the development of novel an-GC approaches, such as targeted therapy that can be applied to suppress cancer development by regulating related gene expression.[10,11] However, more effective and safer targets are still needed. Circular RNAs (circRNAs) are RNA transcripts closed by covalent bonds and participate in human diseases mainly by regulating gene expression rather than encoding proteins,[12, 13] suggesting that circRNAs are promising targets for targeted therapy. CircRNA cRAPGEF5 has been proven to be a potential tumor suppressor in renal cell carcinoma.[14] We performed preliminary RNA-seq analysis and revealed the altered expression of CRAPGEF5 in GC and its inverse correlation with miR-22, 
which is also a critical player in cancer biology.[15] This study was therefore carried out to explore the interaction between CRAPGEF5 and miR-22 in GC.

\section{Methods}

\section{GC patients and tissue acquisition}

A total of 63 patients ( 38 males and 25 females) diagnosed as GC through histopathological exam were enrolled at People's Hospital of Xinjiang Uygur Autonomous Region. The 63 patients included 30 cases at AJCC stage I or II, and 33 cases at AJCC stage III or IV. All cases were adenocarcinoma. All patients signed informed consent. This hospital Ethics Committee approved this study. Age range of patients was 48 to 68 years, with a median of 58 years. Initiated therapy and other severe clinical disorders, such as metabolic disorders, severe infections, other malignancies, were excluded from this study. All patients were diagnosed for the first time, and no recurrent cases were included. Prior to therapy, fine needle aspiration was carried out to collect GC and paired adjacent (with $3 \mathrm{~cm}$ around tumors) non-tumor tissues from the patients. Tissue samples were kept in liquid nitrogen storage prior to the subsequent assays.

\section{GC cells and transfections}

The cell model of GC was AGS (adenocarcinoma) cell line purchased from ATCC (USA). RPMI-1640 medium was mixed with FBS (10\%) to serve as the culture medium of AGS cells. Cells were cultivated in an incubator at $5 \% \mathrm{CO}_{2}, 95 \%$ humidity and $37^{\circ} \mathrm{C}$.

Expression vector of cRAPGEF5 was constructed using pcDNA3.1(+) CircRNA Mini Vector (Addgene). MiR-22 mimic and negative control (NC) miRNA, as well as miR-22 inhibitor and inhibitor NC were purchased from Sigma-Aldrich. AGS cells were cultivated to reach about $80 \%$ confluence, followed by transfecting expression vector of CRAPGEF5 $(1 \mu \mathrm{g})$, miR-22 mimic ( $40 \mathrm{nM})$ or miR-22 inhibitor (40 nM) into $10^{8}$ cells. To perform NC experiments, NC miRNA-, NC inhibitor or empty vector-transfection was performed. Untransfected cells cultivated in fresh medium were also included to serve as a control (C) experiment. Prior to the subsequent experiments, AGS cells were cultivated in fresh medium for further $48 \mathrm{~h}$ in fresh medium.

\section{RNA isolation and quality analysis}

Tissue samples and AGS cells were subjected to total RNA isolations, followed by digesting genomic DNA for $2 \mathrm{~h}$ at $37^{\circ} \mathrm{C}$ using DNase I (Invitrogen). Electrophoresis performed using a $5 \%$ urea-PAGE gel was performed to analyze RNA integrity. Analysis of the purity of RNA samples was performed by measuring the OD 260/280 ratios. RNA samples with an OD 260/280 ratio around 2.0 were pure RNA samples. 


\section{RT-qPCR}

A Reverse Transcription System (A5003, Promega Corporation) was used to synthesize cDNA samples through reverse transcriptions (RTs) with RNA samples as template. With GAPDH as an internal control, qPCRs were performed using a QuantiTect SYBR Green PCR Kit (Qiagen) to analyze the expression of CRAPGEF5. Expression of mature miR-22 and miR-22 precursor was analyzed by RT-qPCR. To measure the expression levels of mature miR-22, poly $(A)$ was added, followed by using poly $(T)$ as reverse primer to perform both RTs and qPCRs. To analyze miR-22 precursor expression, sequence-specific primers were used in RTs and qPCRs U6 was used as the internal control. Three technical replicates were performed for each experiment, and $2^{-\triangle \Delta C T}$ method was used to normalize $C t$ values of target gene to corresponding internal controls.

\section{Cell proliferation assay}

AGS cells with transfections were subjected to proliferation analysis using a CCK-8 kit from SigmaAldrich (USA). Cells were cultivated at $37^{\circ} \mathrm{C}$ in a $96-$ well cell culture plate $(3000$ cells in $0.1 \mathrm{ml}$ fresh medium per well, three replicate wells were set for each experiment), and cell proliferation was analyzed by measuring the OD values at $450 \mathrm{~nm}$ every $24 \mathrm{~h}$ for a total of $96 \mathrm{~h}$. At $2 \mathrm{~h}$ prior to the determination of OD values, CCK- 8 solution was added to reach $10 \%$.

\section{Statistical analysis}

Expression of CRAPGEF5, mature miR-22 and miR-22 precursor in paired tissues was expressed as heatmaps plotted using Heml 1.0 software. Correlations between CRAPGEF5 and mature miR-22 or miR22 precursor were analyzed by Pearson's correlation coefficient. Mean+/-SD values were calculated to express data of multiple cell transfection groups and data comparisons were analyzed by ANOVA and Tukey's test. $P<0.05$ was deemed statistically significant.

\section{Results}

\section{Expression of cRAPGEF5, mature miR-22 and miR-22 precursor was decreased in GC}

Paired GC and non-tumor tissues were collected from GC patients $(n=63)$, followed by measuring the levels of cRAPGEF5, mature miR-22 and miR-22 precursor by performing RT-qPCRs. Expression data were subjected to Heml 1.0 software to express the differential expression of CRAPGEF5, mature miR-22 and miR-22 in GC. Compared to non-tumor tissues, expression levels of cRAPGEF5 (Fig. 1A), mature miR-22

(Fig. 1B) and miR-22 precursor (Fig. 1C) were lower in GC tissues. Therefore, overexpression of cRAPGEF5 and miR-22 may participate in GC. 


\section{CRAPGEF5 was positively correlated with mature miR-22, but not miR-22 precursor across GC tissues}

Correlations between cRAPGEF5 and mature miR-22, or miR-22 precursor across GC tissues were analyzed by performing Pearson's correlation coefficient. It was observed that CRAPGEF5 was positively correlated with mature miR-22 (Fig. 2A), but not miR-22 precursor (Fig. 2B) across GC tissues. Therefore, CRAPGEF5 may affect the maturation of miR-22 in GC.

\section{Overexpression of CRAPGEF5 decreased the expression of mature miR-22 in AGS cells.}

AGS cells were transfected with CRAPGEF5 expression vector or miR-22 mimic to study the relationship between them. Overexpression of CRAPGEF 5 and mature miR-22 was checked every $24 \mathrm{~h}$ for a total of $144 \mathrm{~h}$. It was observed that CRAPGEF5 and mature miR-22 were significantly overexpressed in AGS cells between $48 \mathrm{~h}$ and $144 \mathrm{~h}$ (Fig. 3A, $\mathrm{p}<0.05$ ). Moreover, overexpression of cRAPGEF5 decreased the expression of mature miR-22 between $48 \mathrm{~h}$ and $144 \mathrm{~h}$ (Fig. 3B, p < 0.05), but not miR-22 precursor (Fig. 3C) at each time point. In addition, overexpression of miR-22 did not significantly affect the expression of CRAPGEF5 at each time point (Fig. 3D). Therefore, CRAPGEF5 overexpression may suppress the maturation of miR-22 in AGS cells.

\section{Overexpression of CRAPGEF5 and miR-22 decreased the proliferation of AGS cells}

The effects of CRAPGEF5 and mature miR-22 overexpression as well as miR-22 inhibitor transfection on the proliferation of AGS cells were analyzed by CCK-8 assay. Cell proliferation analysis showed that CRAPGEF5 and miR-22 overexpression decreased cell proliferation, while miR-22 inhibitor played an opposite role. Moreover, miR-22 overexpression attenuated the effect of CRAPGEF5 overexpression (Fig. 4, $\mathrm{p}<0.05)$.

\section{Discussion}

The differential expression of CRAPGEF5 in GC and its functionality have been explored in this study. We found that CRAPGEF5 was under-expressed in GC and may suppress the maturation of miR-22 to inhibit GC cell proliferation.

Based on our knowledge, the functionality of CRAPGEF 5 has only been explored in renal cell carcinoma. It is reported that cRAPGEF5 is under-expressed in renal cell carcinoma and may sponge miR-27a-3p to 
upregulate TXNIP, thereby suppressing the metastasis and growth of tumors.[14] This study is the first to report the downregulation of CRAPGEF5 in GC. Moreover, cell proliferation analysis revealed the inhibitory effects on the proliferation of GC cells. Therefore, CRAPGEF5 may play tumor suppressive roles in GC.

MiR-22 has been reported to be a tumor suppressor in many types of cancers.[15] For instance, miR-22 is downregulated in lung cancer, cervical cancer, prostate cancer, and osteosarcoma, and targets ATP citrate lyase to suppress tumor growth and metastasis. In GC, miR-22 is under-expressed in GC, and overexpression of miR-22 suppresses the invasion and migration of GC cells by targeting Sp1.[16] Consistently, our study also revealed the downregulation of miR-22 in GC and its inhibitory effects on GC cell proliferation. Therefore, our data confirmed the role of miR-22 as a tumor suppressor in GC. However, the upstream regulator of miR-22 in cancer biology remains hardly known.

It has been well established that the main role of circRNAs is to regulate gene expression at transcriptional and translational levels.[12,13] Besides that, circRNAs may also sponge miRNAs to suppress their functions. $[12,13]$ Interesting, our study showed that CRAPGEF5 could promote the maturation of miR-22 in GC cells. Although we failed to explore the in vivo interaction between cRAPGEF5 and miR-22, we observed a significant and positive correlation between them across GC tissues, suggesting the existence of the interaction between them in GC patients. Considering the fact that the maturation of miRNAs requires the transportation of miRNA precursors from nucleus to cytoplasm, cRAPGEF5 may affect the movement of miR-22 precursor to promote its maturation. Our future studies will explore this possibility.

In conclusion, CRAPGEF5 is under-expressed in GC. In addition, CRAPGEF5 may promote the maturation of miR-22 to suppress GC cell proliferation.

\section{Declarations}

\section{Ethics approval and consent to participate}

The study was granted ethical approval by the Ethical Committee of People's Hospital of Xinjiang Uygur Autonomous Region, and all the patients or parents/ guardians of patients provided written informed consent.

\section{Consent for publication}

Not applicable.

\section{Competing interests}

The authors declare that they have no conflicts of interest in this work. 


\section{Funding}

There is no funding to report.

\section{Authors' contribution}

Feng Gao designed the study. Yan Feng carried out experiments and wrote the manuscipt, Feng Gao revised the paper, Chun Wang, Tian Shi, Weidong Liu, Huan Liu and Bo Zhu collected patient specimens and related information. Yan Feng ,Chun Wang, Tian Shi, Weidong Liu, Huan Liu and Bo Zhu contributed to analysing the data. All authors reviewed the results and approved the final version of the manuscript.

\section{Acknowledgements}

The authors would like to thank the participants in this study.

\section{References}

1. Van Cutsem E, Sagaert X, Topal B, Haustermans K, Prenen H: Gastric cancer. Lancet (London, England) 2016, 388(10060):2654-2664.

2. Thrift A, El-Serag H: Burden of Gastric Cancer. Clinical gastroenterology and hepatology : the official clinical practice journal of the American Gastroenterological Association 2020, 18(3):534-542.

3. Datta J, Da Silva E, Kandoth C, Song T, Russo A, Hernandez J, Taylor B, Janjigian Y, Tang L, Solit D et al: Poor survival after resection of early gastric cancer: extremes of survivorship analysis reveal distinct genomic profile. The British journal of surgery 2020, 107(1):14-19.

4. Rawla P, Barsouk A: Epidemiology of gastric cancer: global trends, risk factors and prevention. Przeglad gastroenterologiczny 2019, 14(1):26-38.

5. Tan H, Chia C, Tan G, Choo S, Tai D, Chua C, Ng M, Soo K, Teo M: Metastatic gastric cancer: Does the site of metastasis make a difference? Asia-Pacific journal of clinical oncology 2019, 15(1):10-17.

6. Hallowell B, Endeshaw M, Senkomago V, Razzaghi H, McKenna M, Saraiya M: Gastric cancer mortality rates among US and foreign-born persons: United States 2005-2014. Gastric cancer: official journal of the International Gastric Cancer Association and the Japanese Gastric Cancer Association 2019, 22(5):1081-1085.

7. Duarte H, Gomes J, Machado J, Reis C: Gastric cancer: Basic aspects. Helicobacter 2018:e12523.

8. Ashrafizadeh M, Zarrabi A, Orouei S, Zarrin V, Rahmani Moghadam E, Zabolian A, Mohammadi S, Hushmandi K, Gharehaghajlou Y, Makvandi P et al: STAT3 Pathway in Gastric Cancer: Signaling, Therapeutic Targeting and Future Prospects. Biology 2020, 9(6).

9. Akyala A, Peppelenbosch M: Gastric cancer and Hedgehog signaling pathway: emerging new paradigms. Genes \& cancer 2018, 9:1-10. 
10. Xu W, Yang Z, Lu N: Molecular targeted therapy for the treatment of gastric cancer. Journal of experimental \& clinical cancer research : CR 2016, 35:1.

11. Cetin B, Gumusay O, Cengiz M, Ozet A: Advances of Molecular Targeted Therapy in Gastric Cancer. Journal of gastrointestinal cancer 2016, 47(2):125-134.

12. Patop I, Kadener S: circRNAs in Cancer. Current opinion in genetics \& development 2018, 48:121-127.

13. Zhou R, Wu Y, Wang W, Su W, Liu Y, Wang Y, Fan C, Li X, Li G, Li Y et al: Circular RNAs (circRNAs) in cancer. Cancer letters 2018, 425:134-142.

14. Chen Q, Liu T, Bao Y, Zhao T, Wang J, Wang H, Wang A, Gan X, Wu Z, Wang L: CircRNA cRAPGEF5 inhibits the growth and metastasis of renal cell carcinoma via the miR-27a-3p/TXNIP pathway. Cancer letters 2020, 469:68-77.

15. Xin M, Qiao Z, Li J, Liu J, Song S, Zhao X, Miao P, Tang T, Wang L, Liu W et al: miR-22 inhibits tumor growth and metastasis by targeting ATP citrate lyase: evidence in osteosarcoma, prostate cancer, cervical cancer and lung cancer. Oncotarget 2016, 7(28):44252-44265.

16. Guo M, Hu L, Wang Y, Chen P, Huang J, Lu N, He J, Liao C: miR-22 is down-regulated in gastric cancer, and its overexpression inhibits cell migration and invasion via targeting transcription factor Sp1. Medical oncology (Northwood, London, England) 2013, 30(2):542.

\section{Figures}
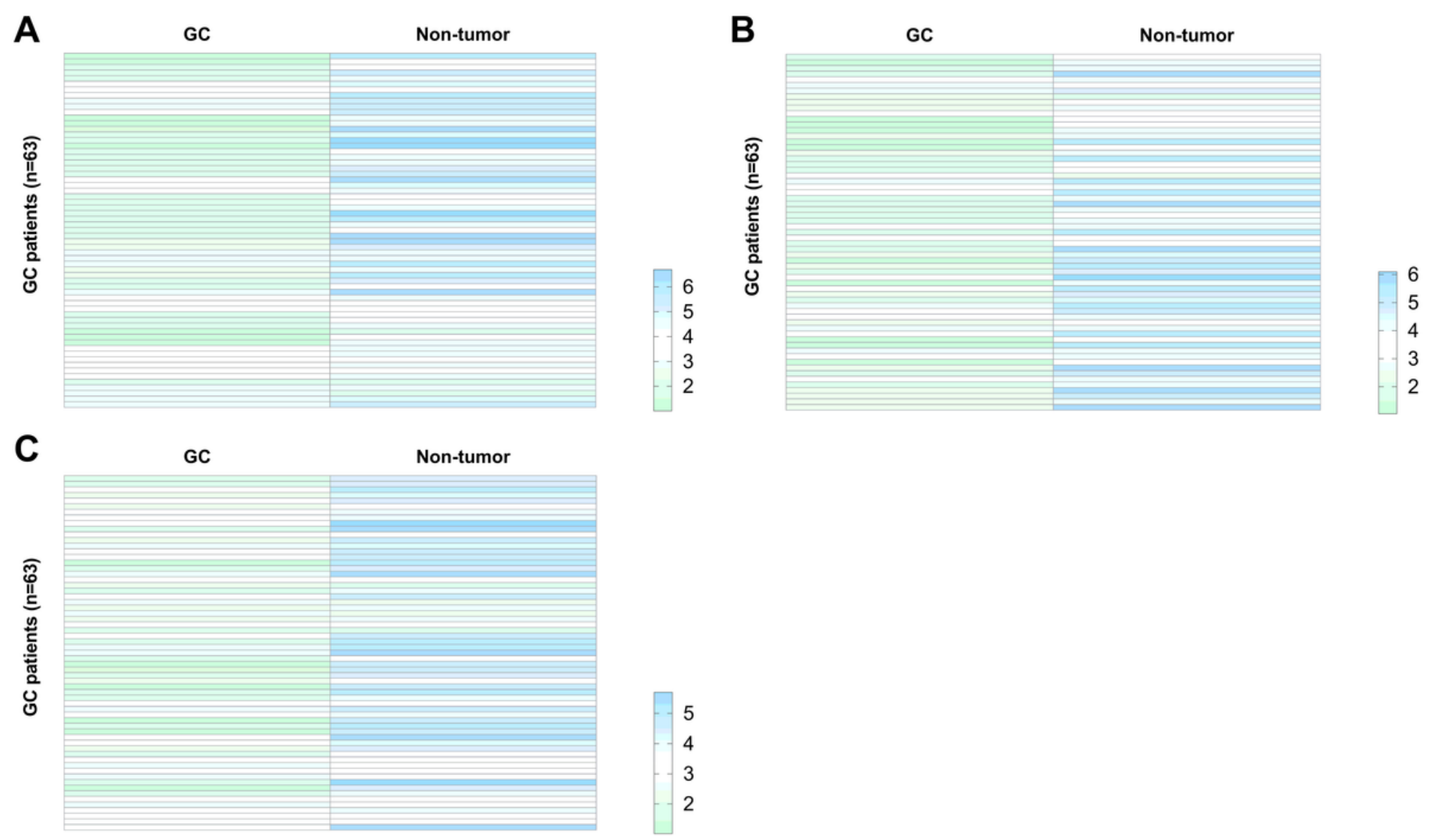

Figure 1 
Expression of cRAPGEF5, mature miR-22 and miR-22 precursor was decreased in GC Paired GC and nontumor tissues were collected from GC patients $(n=63)$, followed by measuring the levels of cRAPGEF5, mature miR-22 and miR-22 precursor by performing RT-qPCRs. Expression data were subjected to Heml 1.0 software to express the differential expression of CRAPGEF5 (A), mature miR-22 (B) and miR-22 (C) in GC.
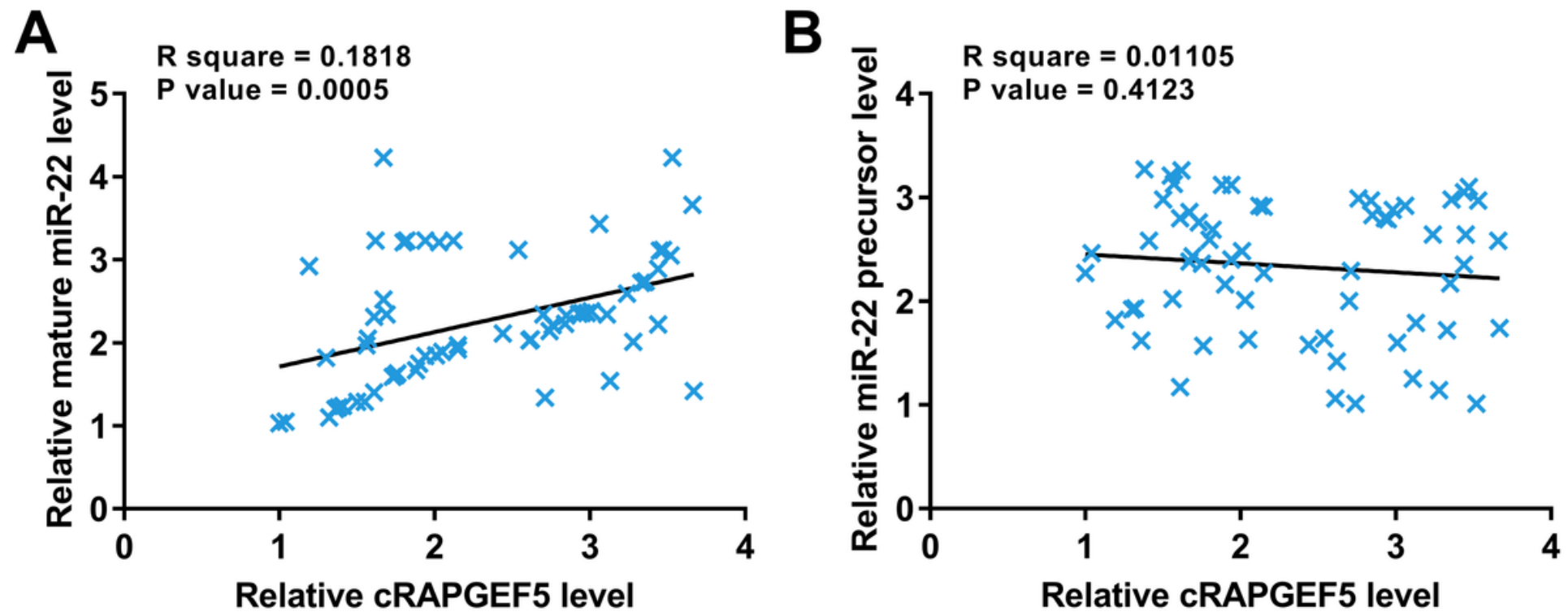

Figure 2

CRAPGEF 5 was positively correlated with mature miR-22, but not miR-22 precursor across GC tissues Correlations between CRAPGEF5 and mature miR-22 (A), or miR-22 precursor (B) across GC tissues were analyzed by performing Pearson's correlation coefficient.

A

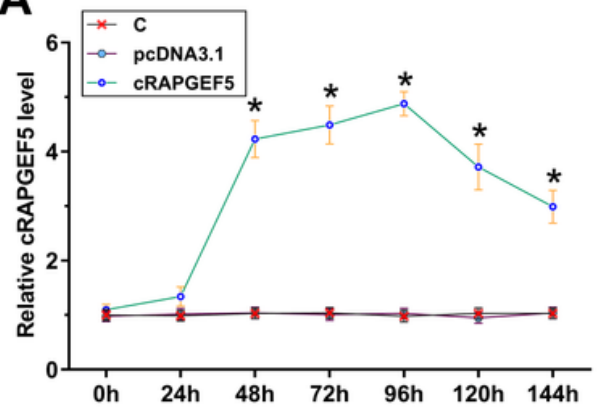

C

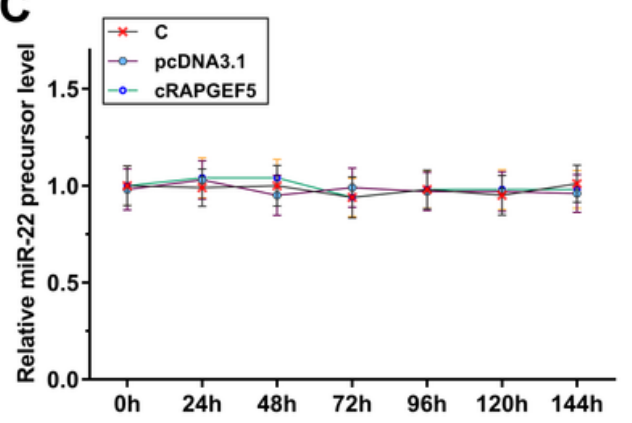

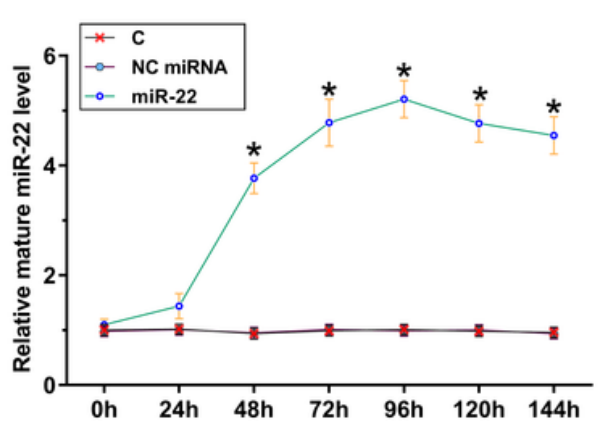

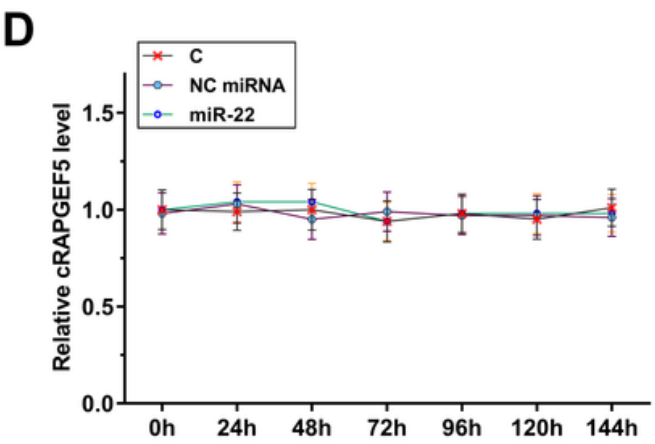

B

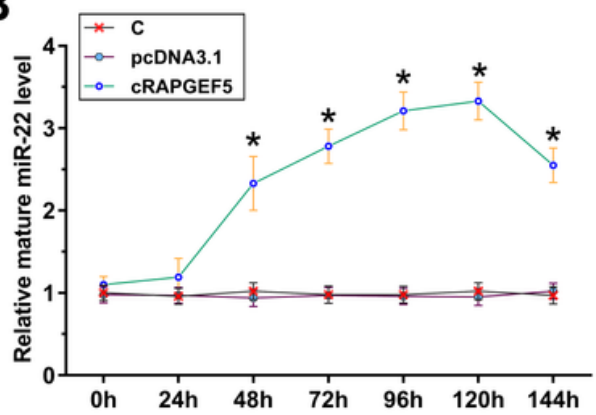

Figure 3 
Overexpression of CRAPGEF5 decreased the expression of mature miR-22 in AGS cells AGS cells were transfected with CRAPGEF5 expression vector or miR-22 mimic to study the relationship between them. Overexpression of cRAPGEF5 and mature miR-22 was checked by RT-qPCR every $24 \mathrm{~h}$ for a total of $144 \mathrm{~h}$ (A). The effects of CRAPGEF5 overexpression on the expression of mature miR-22 (B) and miR-22 precursor (C), and the effects of miR-22 overexpression on CRAPGEF5 expression (D) at each time point were analyzed by RT-qPCR. *, $\mathrm{p}<0.05$.

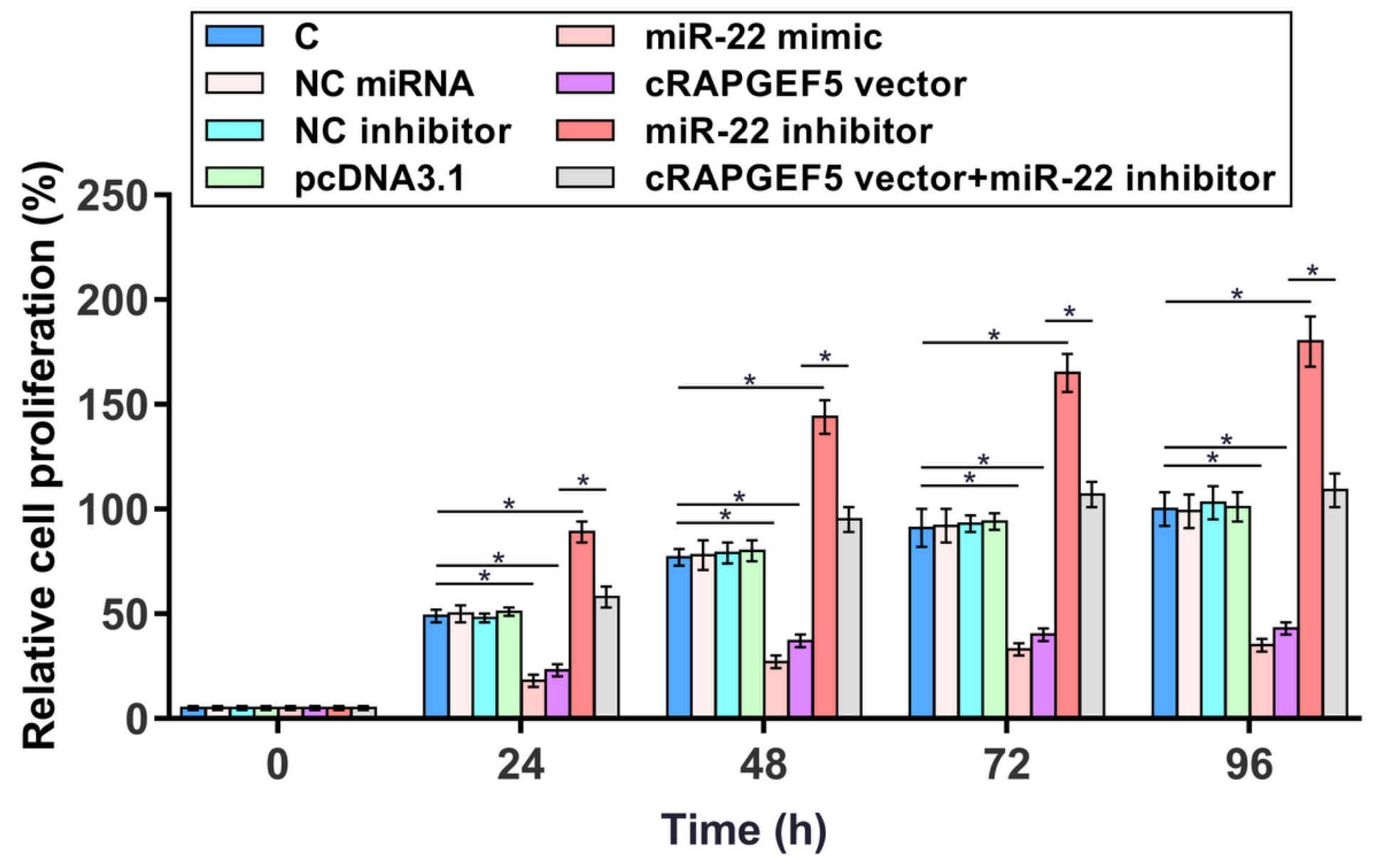

Figure 4

Overexpression of CRAPGEF5 and miR-22 decreased the proliferation of AGS cells The effects of CRAPGEF5 and mature miR-22 overexpression as well as miR-22 inhibitor transfection on the proliferation of AGS cells were analyzed by CCK-8 assay. *, $p<0.05$. 